\title{
The relationship between the surface pressure spectrum and transverse velocity spectrum in a Rapid-distortion theory model of trailing edge noise
}

\author{
M. Z. Afsar* \\ Department of Mechanical \& Aerospace Engineering, University of Strathclyde, Glasgow, G1 I XJ, UK.
}

\begin{abstract}
Accurate jet-surface interaction noise prediction remains an important aspect of the aircraft design process. This is particularly true for the next generation aircraft configurations, one of which could see the exhaust system tightly integrated to the airframe. Use of Rapiddistortion theory of turbulence (RDT) to determine the radiated sound represents one such approach to model the sound generation/propagation process. Recent work on the application of RDT to the canonical problem of a jet flow interacting with a flat plate trailing edge gave accurate predictions across the frequency and acoustic Mach number range. In this paper we ascertain whether an RDT based model that uses the unsteady surface pressure spectrum as the source term can also be utilized to determine accurate edge noise predictions. Surface pressure based models have been widely used in the Amiet formulation of trailing edge noise. The upstream boundary condition in the RDT formulation enters via a streamwise convected quantity, $\tilde{\omega}_{c}\left(\tau-y_{1} / U\left(\boldsymbol{y}_{T}\right), \boldsymbol{y}_{T}\right)$, that is an arbitrary function of its arguments. But since the pressure fluctuation possesses an upstream asymptote that decays algebraically faster than curl of the out-of-plane vorticity fluctuation in the local hydrodynamic relation given by Eq. 3.9 in Goldstein, Leib \& Afsar (J. Fluid Mech., vol. 824, pp. 477-512, 2017), this latter relation cannot be used to determine the surface pressure near the trailing edge. In this paper we show to obtain this relation using an inversion of Fourier transforms similar to that used in our earlier paper Goldstein, Afsar \& Leib (J. Fluid Mech., vol. 736, pp. 532-569, 2013). The relation we obtain shows how the surface pressure spectrum can therefore be related to the velocity fluctuation correlation function.
\end{abstract}

\section{Introduction}

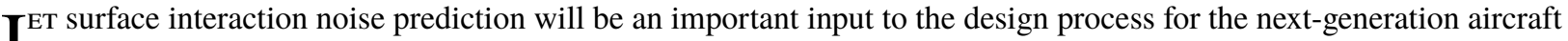
configurations in which the exhaust system is tightly integrated with the airframe [1]. As a consequence, there is much motivation to fund continued research in low-order mathematical models that are capable of predicting the interaction noise for the particular configuration where the external surface is placed very near or completely alligned with the nozzle lower lip line (see Fig. 1). Jet-surface interaction noise refers to the low-frequency amplification of sound that occurs when a high-speed air jet interacts with an external surface. Mathematical models of this phenomenon considers the interaction taking place near the region of discontinuity at the leading or trailing edge of a flat plate that is positioned parallel to the oncoming flow [2]. The noise component caused by the turbulence penetrating into the turbulent boundary layer (i.e. scrubbing noise) is usually neglected. In the main, the analysis is therefore inviscid with the viscous boundary condition relegated to selection of an appropriately unique solution via the application of a Kutta condition at the edge.

Experimentally, the noise amplification associated with jet-surface interaction is fairly significant with several researchers [10] - [18] showing that the presence of a leading/trailing edge results in an increase in sound of about $10 \mathrm{~dB}$ for observation points on the same side as the jet flow. See also experiments at the NASA Glenn Research Center. Bridges et al. [2], Bridges [1] and Zaman et al. [3] determined the power spectral density (PSD) of the far-field pressure for an unheated, high Reynolds number, jet flow emerging from a rectangular nozzle jet flow and interacting with the trailing edge of an external plate. Fig. 12 in Bridges [1], indicates that the difference between the total measured noise (with trailing edge) and the isolated jet noise is greater at lower acoustic Mach numbers. This effect is more prominent when the distance from the plate trailing edge location to nozzle exit plane is increased (see Fig. 8 in [2] and also p.299 and Fig. 11a in [4]).

\footnotetext{
*Chancellor's Fellow. AIAA Member.
} 
There has been a lot of recent work using Rapid-distortion theory (RDT) to determine the scattered sound field for the canonical problem of a jet flow interacting with a trailing edge described in the Bridges et al. experiments above. The upstream boundary condition enters in the RDT formulation via a streamwise convected quantity, $\tilde{\omega}_{c}\left(\tau-y_{1} / U\left(\boldsymbol{y}_{T}\right), \boldsymbol{y}_{T}\right)$, that is an arbitrary function of its arguments. Goldstein, Afsar \& Leib ([5], hereafter referred to as GAL) obtained a formula for the acoustic spectrum that performed reasonably well against the experimental data. The convected quantity, tilde $\omega_{c}$ was found by inverting the formula for the spanwise Fourier transform of the transverse velocity fluctuation. An algebraic relationship connecting the spectrum of $\tilde{\omega}_{c}$ and the former velocity correlation was then obtained in the flow over a doubly infinite streamwise/spanwise surface. Although there were a number of technical issues with this approach. (1). this relation does not have the consistency requirements that one would ideally expect of an boundary condition since it is determined in the region of scattered flow. As mentioned, numerically the model gave reasonable predictions in the main. However, Afsar et al. ([6], referred to as ALB) showed that at very low frequencies the GAL acoustic spectrum formula tends to an $O(1)$ constant. Inclusion of so-called anti-correlation effects in the model for the auto-covariance of the transverse velocity fluctuation remedied this defect by showing that the acoustic spectrum behaved like a dipole (i.e. $O\left(\omega^{2}\right)$, where $\omega$ is the angular frequency) as $\omega \rightarrow 0$. In the latter paper several different trailing edge locations were used and the jet mean flow and turbulent kinetic energy profiles were obtained via a Reynolds Averaged Navier Stokes (RANS) solution.

A more consistent relation for $\omega_{c}$ was obtained later by Goldstein, Leib \& Afsar (GLA, [7]) who analyzed the far-upstream behaviour of a local evolution equation for this quantity. The rationale for this is that in the RDT limit the interaction scale and the shear layer growth scale (over which an eddy may turnover) are asymptotically disparate when the interaction time for the distortion is short and the turbulence intensity is also a small parameter. This gave a formula for $\omega_{c}$ that apriori relates it to the hydrodynamic field measured by an experimentalist that then serves as the input to the scattering problem. De-correlation effects were also included in this model (see their Eqs. 6.8 \& 6.13). The resulting predictions were found to be robust down to very low frequencies.

In this paper our aim is to show how to relate $\omega_{c}$ to the surface pressure spectrum. Use of the latter is popular in Amiet-type models. We show that the local relation derived in reference [7] cannot be used in this context because of the fact that the pressure fluctuation decays (and drops out of the lowest order expansion) in the basic evolution equation for $\tilde{\omega}_{c}$. Recourse is then made to a Fourier inversion approach. The aim of this paper is to ascertain what impact this formula (provided shortly and derived in the full paper) has on the predictive capability of the RDT model.

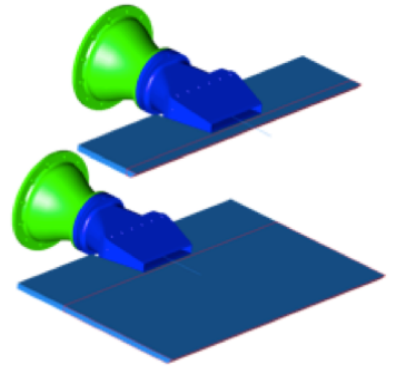

(a) Plate flush with nozzle lower lip.

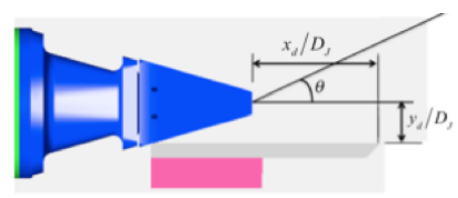

(b) Geometric arrangement

Fig. 1 Nozzle/plate configuration for plate close to nozzle lip (courtesy Dr. James E. Bridges, NASA Glenn)

\section{Summary of previous results: GAL, ALB and GLA}

The turbulent exhaust flow emerging from the rectangular nozzle in Fig.[?? interacts with the trailing edge to produce a highly amplified noise source. The sound radiation is given by the the far-field limit of the pressure fluctuation field that is governed by the linearized Navier-Stokes equations. However the latter set of equations reduce in complexity when the realization that the maximum sound is generated during the interaction. In this case Rapid-distortion theory 
(RDT) may be used. The premise of which is that linear analysis describes the interaction of turbulence with solid surfaces. RDT applies when the turbulence intensity is taken as a global small parameter everywhere in the flow; i.e. $\alpha=\left|\boldsymbol{u}^{\prime}\right| / U_{J} \ll O(1)$ where $\alpha$ is the turbulence intensity, $\left|\boldsymbol{u}^{\prime}\right|$ is the magnitude of the local rms turbulence velocity and $U_{J}$ is the nozzle exit velocity. It also implies that the length (or time) scale over which the interaction takes place is short compared to the length (or time) scale over which the turbulent eddies evolve. Hence, when interpreted asymptotically, GLA explain how these assumptions imply, that it is possible to identify a distance that is very (infinitely) large on the scale of the interaction, but still small on the scale over which the turbulent eddies evolve. Hence the interacting flow can be taken as inviscid and non-heat-conducting. It is, therefore, governed by the linearized Euler equations linearized about an arbitrary base flow. Hence, RDT can be thought of as the lowest order perturbation of the Navier-Stokes equations in the small turbulence intensity, $\alpha \ll O(1)$, in the vicinity of the trailing edge. The interaction problem is, therefore, linear and inviscid and the mean flow near the trailing edge is nearly transversely sheared. In other words, when the flow Reynolds number is large, $R=U_{J} D_{J} / v$ (where $v$ is the kinematic viscosity) and the turbulence interaction Reynolds number $R_{T}$ is order-1. Hence $R_{T}=\alpha R=O(1)$ where $\left(U_{J}, D_{J}\right)$ are appropriate reference velocity and length scales respectively.

Using Green's theorem and (2.4), (2.7), (2.8), (2.11)-(2.14) in GAL, GLA show that the lineaized Euler equations possess basic solutions for the inviscid pressure perturbation, $p^{\prime}(\boldsymbol{x}, t)=(p-\bar{p})(\boldsymbol{x}, t)$ and mass flux, $u_{i} \equiv \rho v_{i}^{\prime}(\boldsymbol{x}, t)$ (where $v_{i}^{\prime}$ denotes the vector velocity perturbation) given by

$$
p^{\prime}(\boldsymbol{x}, t)=\frac{D_{0}^{3}}{D t^{3}} \int_{-T}^{T} \int_{V_{\infty}(\boldsymbol{y})} g(\boldsymbol{y}, \tau \mid \boldsymbol{x}, t) \tilde{\omega}_{c}\left(\tau-y_{1} / U\left(\boldsymbol{y}_{T}\right), \boldsymbol{y}_{T}\right) d \boldsymbol{y} d \tau,
$$

and

$$
u_{\perp}^{\prime}(\boldsymbol{x}, t)=-\frac{\partial U / \partial y_{i}}{|\boldsymbol{\nabla} U|} \int_{-T}^{T} \int_{V_{\infty}(\boldsymbol{y})} g_{i}(\boldsymbol{y}, \tau \mid \boldsymbol{x}, t) \tilde{\omega}_{c}\left(\tau-y_{1} / U\left(\boldsymbol{y}_{T}\right), \boldsymbol{y}_{T}\right) d \boldsymbol{y} d \tau,
$$

respectively. $T$ denotes a very large, but finite, time interval and $\bar{p}$ is the time-averaged pressure field (Favre averages are used for velocity and temperature). The surface $S(\boldsymbol{y})$ bound volume $V(\boldsymbol{y})$ in $(1) \&(2)$ can be finite, semi-infinite or infinite in the streamwise direction but its generators must be parallel to the level curves of the mean velocity field, $U\left(\boldsymbol{y}_{T}\right)=$ const. The Green's function, $g_{i}(\boldsymbol{y}, \tau \mid \boldsymbol{x}, t)$, is linearly related to direct Rayleigh Green's function $g(\boldsymbol{y}, \tau \mid \boldsymbol{x}, t)$, which is determined for incoming wave behavior as $|\boldsymbol{y}| \rightarrow \infty$ and appropriate boundary conditions on $S(\boldsymbol{y})$. The convective derivative in (1) is $D_{0} / D t=\partial / \partial t+U\left(\boldsymbol{x}_{T}\right) \partial / \partial x_{1}$ and mean flow gradient in (2) is given by $\partial U\left(\boldsymbol{y}_{T}\right) / \partial y_{i}$ where $\boldsymbol{x}=\left\{x_{1}, \boldsymbol{x}_{T}\right\}=\left\{x_{1}, x_{2}, x_{3}\right\}$ and $\boldsymbol{y}=\left\{y_{1}, \boldsymbol{y}_{T}\right\}=\left\{y_{1}, y_{2}, y_{3}\right\}$.

\section{A. Acoustic spectrum formula derived by GLA}

As mentioned in $\S .1$, since $\tilde{\omega}_{c}\left(\tau-y_{1} / U\left(\boldsymbol{y}_{T}\right), \boldsymbol{y}_{T}\right)$ satisfies $D_{0} \tilde{\omega}_{c} / D \tau=0$ by definition, for an arbitrary selection of its arguments, $\left(\tau-y_{1} / U\left(\boldsymbol{y}_{T}\right), \boldsymbol{y}_{T}\right)$, it can be used to specify the upstream boundary condition (i.e. the input) within a boundary value problem (BVP) that seeks to determine acoustic spectrum as its output. GLA showed that the output in this case, i.e. acoustic spectrum of the scattered pressure, $2 \pi I(\boldsymbol{x}, \omega)=\int_{-\infty}^{\infty} p^{(s)}(\boldsymbol{x}, t) \widetilde{p^{(s) *}}(\boldsymbol{x}, t+\tau) d \tau$, where $\tilde{\boldsymbol{\bullet}}$ denotes Favre average of $\bullet, *$ is complex conjugate and $p^{(s)}(\boldsymbol{x}, t)$ is the scattered part of the pressure fluctuation. GLA show that when the mean flow is planar, i.e. $U\left(y_{2}\right), I(\boldsymbol{x}, \omega)$ is given by

$$
I(\boldsymbol{x}, \omega) \sim \int_{0}^{\infty} \int_{0}^{\infty} D\left(y_{2}, \tilde{y}_{2} ; \theta\right) Q\left(y_{2} ; \omega, \theta\right) Q^{*}\left(\tilde{y}_{2} ; \omega, \theta\right) S\left(y_{2}, \tilde{y}_{2} ; k_{3}^{*}, \omega\right) d y_{2} d \tilde{y}_{2},
$$

where $D\left(y_{2}, \tilde{y}_{2} ; \theta\right)$ is a planar-flow directivity factor determined by application of the Wiener-Hopf technique (see $\S .6$ in GAL) The parameters $(\omega, \theta)$ are the angular frequency and polar observation angle with respect to the jet centerline. $S\left(y_{2}, \tilde{y}_{2} ; k_{3}^{*}, \omega\right)$ is the space-time Fourier transform of the auto-covariance of $\tilde{\omega}_{c}\left(\tau-y_{1} / U\left(\boldsymbol{y}_{T}\right), \boldsymbol{y}_{T}\right)$. GLA [1] show that this latter quantity is directly related to the auto-covariance of the transverse velocity correlation $R_{22}$ via Eqs. (6.4), (6.6), (6.8), (6.9) \& (6.13) in their paper. They use this direct algebraic correspondence of $S\left(y_{2}, \tilde{y}_{2} ; k_{3}^{*}, \omega\right)$ to $R_{22}$ determine $S$ since accurate models of $R_{22}$ can be constructed [6]. The parameter $k_{3}^{*}$ is the spanwise wavenumber evaluated at the 
stationary phase point where the latter is determined by sequentially evaluating the streamwise and spanwise inverse Fourier transforms of the propagator function $\mathcal{R}$ in (6.14) \& (6.19) in GAL.

The remaining term in (3), $Q\left(y_{2} ; \omega, \theta\right)$, is determined by using the high frequency WKBJ approximation of the homogeneous Rayleigh equation in the form of $L P^{>}=0$ where $P^{>}$represents the appropriate outgoing wave solution to the homogeneous form of (A.3) in GAL. GLA constructed this using the first term of asymptotic series in inverse wavenumber $\left(k_{\infty}^{-1}\right)$. They found that $Q\left(y_{2} ; \omega, \theta\right)=1$ as $k_{\infty}<<O(1)$ and is bounded by an transcendentally small term of size $O\left(e^{-k_{\infty} y_{2}}\right)$ at large $y_{2}$ and is $o(1)$ at $y_{2}=O(1)$ when the far-field wavenumber gets large as, $k_{\infty}=\omega / c_{\infty} \rightarrow \infty$ ( $c_{\infty}$ is the speed of sound).

\section{Example results from previous studies}

In Fig. [??] we reproduce the $90^{\circ}$ spectrum results for the experiments reported in Bridges (2014) using the ALB and GLA models. The trailing edge location is indicated in the figure caption. The former ALB result is compared to the GAL model also (dashed curve in Fig. ?? a). It is clear that the while the ALB predictions look reasonable up to a Strouhal number of 0.5 , the GLA results show much closer agreement at low frequencies and extend to $S t \approx 0.7$.

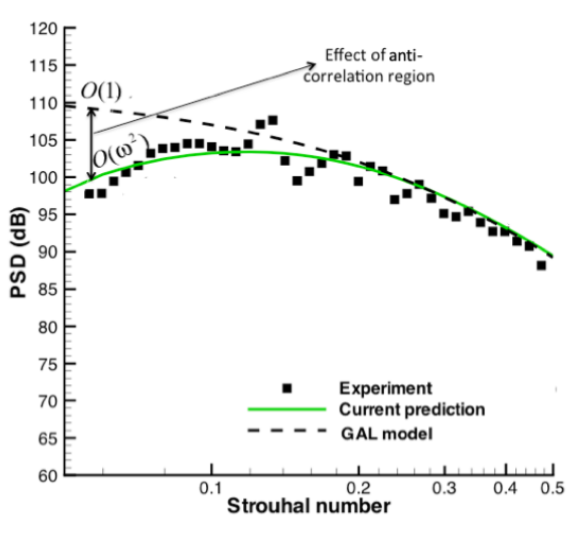

(a) Fig. 5 b in Afsar, Leib \& Bozak [6]

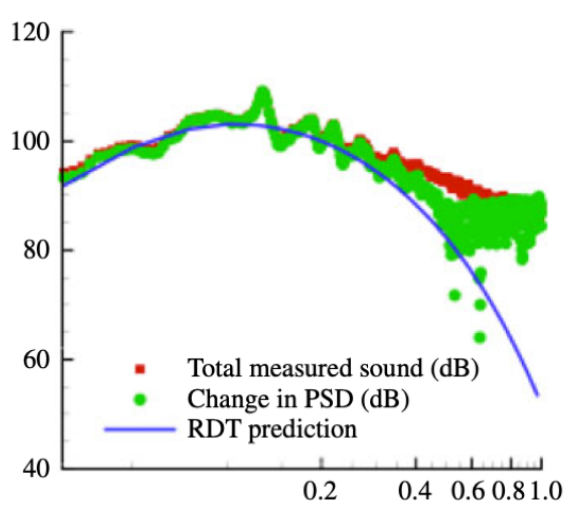

(b) Fig. 8a in Goldstein, Leib \& Afsar [7]

Fig. 2 Power Spectral Density (PSD) of the far-field pressure fluctuations at 100 equivalent diameters from nozzle exit (lossless in $\mathbf{~ d B}$ scale referenced to $20 \mu \mathrm{Pa}$ ) as a function of Strouhal number for $M a=0.9$. Plate trailing edge at $y_{d} / D_{J}=1.1, x_{d} / D_{J}=5.7$, where $D_{J}=2.12^{\prime \prime}, \psi=-90$ and $\theta=90^{\circ}$.

\section{Surface pressure based boundary condition for $\tilde{\omega}_{c}$}

To fix ideas we consider the low frequency form of (3) where $Q\left(y_{2} ; \omega, \theta\right)=1$. That is:

$$
I(\boldsymbol{x}, \omega) \sim \int_{0}^{\infty} \int_{0}^{\infty} D\left(y_{2}, \tilde{y}_{2} ; \theta\right) S\left(y_{2}, \tilde{y}_{2} ; k_{3}^{*}, \omega\right) d y_{2} d \tilde{y}_{2},
$$

In the paper we show that the spanwise space-time Fourier transform of $\tilde{\omega}_{c}$,

$$
\hat{\Omega}\left(y_{2} ; k_{3}, \omega\right) \equiv \frac{1}{(2 \pi)^{2}} \int_{-\infty}^{\infty} \int_{-\infty}^{\infty} e^{i\left(\omega z-y_{3} k_{3}\right)} \tilde{\omega}_{c}\left(z, y_{2}, y_{3}\right) d z d y_{3}=\frac{1}{(2 \pi)} \int_{-\infty}^{\infty} e^{-i y_{3} k_{3}} \Omega\left(y_{2}, y_{3} ; \omega\right) d y_{3},
$$

can be shown to be related to the spanwise transform of the pressure fluctuation by the following formula

$$
\hat{\Omega}\left(y_{2} ; k_{3}, \omega\right)=\frac{\omega\left|U^{\prime}\left(y_{2}\right)\right|}{(2 \pi)^{4} U^{2}\left(y_{2}\right)} \frac{\int_{-\infty}^{\infty} e^{-i x_{3} k_{3}} \hat{P}\left(0, x_{3} ; \omega / U\left(y_{2}\right), \omega\right) d x_{3}}{G\left(y_{2} \mid 0 ; \omega, \omega / U\left(y_{2}\right), k_{3}\right)},
$$


where $\hat{P}\left(0, x_{3} ; \omega / U\left(y_{2}\right), \omega\right)$ is evaluated on the plate surface, $x_{2}=0$, in the temporal/streamwise Fourier transform of the pressure fluctuation

$$
\hat{P}\left(x_{2}, x_{3} ; k_{1}, \omega\right)=\frac{1}{(2 \pi)^{2}} \int_{-\infty}^{\infty} \int_{-\infty}^{\infty} e^{i\left(\omega t-k_{1} x_{1}\right)} p^{\prime}\left(x_{1}, x_{2} x_{2}, t\right) d t d x_{1}=\frac{1}{(2 \pi)} \int_{-\infty}^{\infty} e^{-i k_{1} x_{1}} P\left(x_{2} x_{3} ; \omega\right) d x_{1},
$$

and $G\left(y_{2} \mid 0 ; \omega, \omega / U\left(y_{2}\right), k_{3}\right)$ is the scaled Rayleigh equation Green's function given by solution (A.4) \& (A.5) to equation (A.3) in GAL.

The formula (6) was obtained by the Fourier inversion method used in GAL. Since its auto-covariance must be equivalent to (5.7) in GAL, it implies that the spanwise transform of the transverse mass flux $\rho V_{\perp}$ is related to the surface pressure fluctuation via the following

$$
\int_{-\infty}^{\infty} e^{-i x_{3} k_{3}} \rho V_{\perp}\left(0, x_{3} ; \omega / U\left(y_{2}\right), \omega\right) d x_{3} \sim \mathcal{K}\left(y_{2} ; \omega / U\left(y_{2}\right), \omega\right) \int_{-\infty}^{\infty} e^{-i x_{3} k_{3}} \hat{P}\left(0, x_{3} ; \omega / U\left(y_{2}\right), \omega\right) d x_{3}
$$

where $\mathcal{K}\left(y_{2} ; \omega / U\left(y_{2}\right), \omega\right)$ is the transfer function

$$
\mathcal{K}\left(y_{2} ; \omega / U\left(y_{2}\right), \omega\right) \equiv \frac{G_{2}\left(y_{2} \mid x_{2}^{(1)} ; \omega, \omega / U\left(y_{2}\right), k_{3}\right)}{G\left(y_{2} \mid 0 ; \omega, \omega / U\left(y_{2}\right), k_{3}\right)},
$$

$x_{2}^{(1)}$ is transverse location where $U\left(x_{2}\right)$ is maximum (see $\S .6$ of GAL) and $G_{2}$ is the streamwise/temporal Fourier transform of the transverse component of (3.11) in GAL.

The relationship between the spanwise spectra of transverse mass flux and that for the surface pressure fluctuation can be easily obtained from (8). Using the same notation as $\$ .6$ in GAL, we find that the spectrum of spanwise spatial and temporal Fourier transform $F_{\perp}$

$$
F_{\perp}\left(x_{2}, \tilde{x}_{2} \mid y_{2}, \tilde{y}_{2} ; \omega, k_{3}\right) \equiv \frac{1}{(2 \pi)^{2}} \int_{-\infty}^{\infty} \int_{-\infty}^{\infty} e^{-i\left(\omega \tau-k_{3} \eta_{3}\right)} f_{\perp}\left(x_{2}, \tilde{x}_{2} \mid \omega / U\left(y_{2}\right), \omega / U\left(\tilde{y}_{2}\right) ; \eta_{3}, \tau\right) d \eta_{3} d \tau
$$

related to the physically measureable quantity $f_{\perp}$ :

$$
f_{\perp}\left(x_{2}, \tilde{x}_{2} \mid y_{2}, \tilde{y}_{2} ; \omega, k_{3}\right) \equiv \frac{1}{(2 \pi)^{2}} \int_{-\infty}^{\infty} \int_{-\infty}^{\infty} e^{-i\left(x_{1} k_{1}-\tilde{x}_{1} \tilde{k}_{1}\right)}<\rho v_{\perp}^{(0)}\left(x_{1}, x_{2}, x_{3}, t\right) \rho v_{\perp}^{(0)}\left(\tilde{x}_{1}, \tilde{x}_{2}, \tilde{x}_{3}+\eta, t+\tau\right)>d x_{1} d \tilde{x}_{1},
$$

where $\langle\ldots\rangle$ is a spanwise measure of the ensemble average

$$
<\rho v_{\perp}^{(0)}\left(x_{1}, x_{2}, x_{3}, t\right) \rho v_{\perp}^{(0)}\left(\tilde{x}_{1}, \tilde{x}_{2}, \tilde{x}_{3}+\eta, t+\tau\right)>=\lim _{T \rightarrow \infty} \int_{-\infty}^{\infty} \int_{-\infty}^{\infty} \rho v_{\perp}^{(0)}\left(x_{1}, x_{2}, x_{3}, t\right) \rho v_{\perp}^{(0)}\left(\tilde{x}_{1}, \tilde{x}_{2}, \tilde{x}_{3}+\eta, t+\tau\right) d t d x_{3},
$$

is related to surface-pressure spectrum function:

$$
H_{\perp}\left(0,0 \mid y_{2}, \tilde{y}_{2} ; \omega, k_{3}\right) \equiv \frac{1}{(2 \pi)^{2}} \int_{-\infty}^{\infty} \int_{-\infty}^{\infty} e^{-i\left(\omega \tau-k_{3} \eta_{3}\right)} h_{\perp}\left(0,0 \mid \omega / U\left(y_{2}\right), \omega / U\left(\tilde{y}_{2}\right) ; \eta_{3}, \tau\right) d \eta_{3} d \tau,
$$

via

where $h_{\perp}$ is the physically measureable surface pressure statistical quantity :

$$
h_{\perp}\left(0,0 \mid y_{2}, \tilde{y}_{2} ; \omega, k_{3}\right) \equiv \frac{1}{(2 \pi)^{2}} \int_{-\infty}^{\infty} \int_{-\infty}^{\infty} e^{-i\left(x_{1} k_{1}-\tilde{x}_{1} \tilde{k}_{1}\right)}<p^{(0)}\left(x_{1}, 0, x_{3}, t\right) p^{(0)}\left(\tilde{x}_{1}, 0, \tilde{x}_{3}+\eta, t+\tau\right)>d x_{1} d \tilde{x}_{1},
$$


in which $\langle\ldots>$ in $(14)$ is also a spanwise measure but now of the ensemble average of the surface pressure

$$
<p^{(0)}\left(x_{1}, 0, x_{3}, t\right) p^{(0)}\left(\tilde{x}_{1}, 0, \tilde{x}_{3}+\eta, t+\tau\right)>=\lim _{T \rightarrow \infty} \int_{-\infty}^{\infty} \int_{-\infty}^{\infty} p^{(0)}\left(x_{1}, 0, x_{3}, t\right) p^{(0)}\left(\tilde{x}_{1}, 0, \tilde{x}_{3}+\eta, t+\tau\right) d t d x_{3},
$$

The relation between (10) and (13) (i.e. between the density-weighted transverse velocity correlation function and the surface pressure) is then given by:

$$
F_{\perp}\left(x_{2}, \tilde{x}_{2} \mid y_{2}, \tilde{y}_{2} ; \omega, k_{3}\right) \sim \mathcal{K}\left(y_{2} ; \omega / U\left(y_{2}\right), \omega\right) \mathcal{K}^{*}\left(\tilde{y}_{2} ; \omega / U\left(\tilde{y}_{2}\right), \omega\right) H_{\perp}\left(0,0 \mid y_{2}, \tilde{y}_{2} ; \omega, k_{3}\right)
$$

where $\mathcal{K}$ is given by 9 . The spectrum of $\tilde{\omega}_{c}$,

$$
\begin{aligned}
S\left(y_{2}, \tilde{y}_{2} ; k_{3}, \omega\right) & =(2 \pi)^{2} \lim _{T \rightarrow \infty} \frac{\hat{\Omega}\left(y_{2}, k_{3}, \omega, T\right) \hat{\Omega}^{*}\left(y_{2}, k_{3}, \omega, T\right)}{2 T} \\
& :=\frac{1}{(2 \pi)} \int_{-\infty}^{\infty} \int_{-\infty}^{\infty} e^{i\left(\omega \tau-k_{3} \eta_{3}\right)}<\tilde{\omega}_{c}\left(t, y_{2}, y_{3}\right) \tilde{\omega}_{c}\left(t+\tau, \tilde{y}_{2}, y_{3}+\eta_{3}\right)>d \eta_{3} d \tau
\end{aligned}
$$

is given by

$$
S\left(y_{2}, \tilde{y}_{2} ; k_{3}, \omega\right)=\frac{\omega^{2}\left|U^{\prime}\left(y_{2}\right) U^{\prime}\left(\tilde{y}_{2}\right)\right|}{(2 \pi)^{6} U^{2}\left(y_{2}\right) U^{2}\left(\tilde{y}_{2}\right)} \frac{H_{\perp}\left(0,0 \mid y_{2}, \tilde{y}_{2} ; \omega, k_{3}\right)}{G\left(y_{2} \mid 0 ; \omega, \omega / U\left(y_{2}\right), k_{3}\right) G^{*}\left(\tilde{y}_{2} \mid 0 ; \omega, \omega / U\left(\tilde{y}_{2}\right), k_{3}\right)} .
$$

Alternatively, (18) can be expressed in terms of the spectrum of the transverse velocity correlation using (10, , 11), (12), (5.7) in GAL \& 9] as follows

$$
\left.S\left(y_{2}, \tilde{y}_{2} ; k_{3}, \omega\right)\right|_{\text {Eq. } 5.7 \text { in GAL }}=\left.\left[\mathcal{K}\left(y_{2} ; \omega / U\left(y_{2}\right), \omega\right) \mathcal{K}^{*}\left(\tilde{y}_{2} ; \omega / U\left(\tilde{y}_{2}\right), \omega\right)\right] S\left(y_{2}, \tilde{y}_{2} ; k_{3}, \omega\right)\right|_{18} .
$$

\section{Conclusions and future work}

The source term in recent Rapid-distortion theory models of trailing edge noise is the convected quantity, $\tilde{\omega}_{c}\left(\tau-y_{1} / U\left(\boldsymbol{y}_{T}\right), \boldsymbol{y}_{T}\right)$, that is an arbitrary function of its arguments. The aim of this paper is to express the spectrum of $\tilde{\omega}_{c}$ in terms of surface pressure near the trailing edge (as opposed to the transverse velocity correlations used in the models above). Surface pressure based models have been widely used in the Amiet formulation of trailing edge noise. We found a relation connecting the spectrum of $\tilde{\omega}_{c}$ to surface pressure spectrum, (18) and also a formula relating this latter term to that obtained by using the transverse velocity correlation function (see Eqs. 16 \& 19]. The analysis remaining for this paper will include a full derivation and discussion of Eq. 6). As stated, this is the key relation in this paper because it expresses the spanwise space-time Fourier transform of $\tilde{\omega}_{c}$ to the same spanwise transforms of the surface pressure (i.e. the spanwise space-time Fourier transform of pressure fluctuation evaluated at the plate surface). The critical approximation in this relation is the transverse location where the latter Fourier transform is evaluated. The GAL theory shows that this type of transform (the transverse velocity was considered in that paper in the form of their Eq. 4.14) should be evaluated at the point where $U\left(x_{2}\right)$ is maximum. However, experimental data in Scharton et al. [8] explains how this particular transform remains relatively invariant to tranverse location. In the full paper we discuss the implications of this and also numerically evaluate (13) using the model in GAL for the transverse velocity spectrum (10). The aim will be to test whether accurate predictions are possible when one uses the surface pressure as the effective source model in the Rapid-distortion theory calculation of low frequency jet-surface interaction noise.

\section{Acknowledgments}

MZA would like to thank Strathclyde University for financial support from the Chancellor's Fellowship.

\section{References}

[1] Bridges, J. E., "Noise from Aft Deck Exhaust Nozzles - Differences in Experimental Embodiments," 21st AIAA/CEAS Aeroacoustics Conference, 2014. 
[2] Bridges, J. E., Brown, C. A., and Bozak, R. F., "Experiments on exhaust noise of tightly integrated propulsion systems," 21st AIAA/CEAS Aeroacoustics Conference, 2014.

[3] Zaman, K. B. M. Q., Brown, C. A., and Bridges, J. E., "Interaction of a Rectangular Jet with a Flat-plate Placed Parallel to the Flow," 20th AIAA/CEAS Aeroacoustics Conference, 2013.

[4] Wang, M. E., “Wing effect on jet noise propagation,” J. Aircraft, Vol. 18, 1981, p. 295 - 302.

[5] Goldstein, M. E., Afsar, M. Z., and Leib, S. J., "Non-homogeneous Rapid-distortion theory on transversely sheared flows," J. Fluid Mech., Vol. 736, 2013, pp. 532-569.

[6] Afsar, M. Z., Leib, S. J., and Bozak, R. E., "Effect of de-correlating turbulence on the low frequency decay of jet-surface interaction noise in sub-sonic unheated air jets using a CFD-based approach," J. Sound and Vib., Vol. 386, 2017, pp. 177-207.

[7] Goldstein, M. E., Leib, S. J., and Afsar, M. Z., "Generalized rapid distortion theory on transversely sheared mean flows with physically realizable upstream boundary conditions: application to the trailing edge problem," J. Fluid Mech., Vol. 824, 2017, pp. 477-512.

[8] Scharton, T. D., Pinkel, B., and Wilby, J. F., "A study of trailing edge blowing as a means of reducing noise generated by the interaction of flow with a surface," NASA CR, Vol. 18, 1981, pp. NASA CR-132270. 